\title{
MATHEMATICAL FOUNDATIONS OF CARTOGRAPHY
}

The aim of this contribution is to acquaint the reader with a specific part of mathematical cartography that is interesting not only from the point of view of mathematics, but also from the point of view of constructive geometry.

\section{Introduction}

The surface of the Earth is quite irregular and complex which makes it difficult to represent in models. Despite significant progress in geographic information systems which give us more detailed and more precise view of the landscape surrounding us, the map remains the most common form of representation. In many domains of human activity, maps are one of the key tools without which we cannot imagine work of experts, nor even our leisure time.

\section{Mathematical cartography}

The purpose of cartography is construction of maps of the Earth and its parts, the so-called geodetic systems, on which the surface of the Earth is depicted together with real objects and phenomena. Map construction is a process based on a geometrical transformation. Therefore, geometry plays an important role in cartography. It allows us to mathematically describe cartographic transformations enabling us to rigorously study properties of cartographic projections.

The methods of representing geodetic systems in the plane are the primary concern of mathematical cartography. Namely, mathematical cartography is that part of cartography which is concerned with mathematical and geometrical fundamentals of cartography in the general sense of the word. It studies processes of transformation of space coordinates of objects and phenomena on surfaces of reference to the surface of a map. It is concerned with calculations and constructions used for transforming the curved surface of the Earth to the plane through cartographical projections. Therefore, mathematics and geometry form the theoretical basis for cartography.

\section{Classification of cartographic projections}

We consider the reference surface of the Earth to be the geometrical surface that is a mathematical model of the Earth. In practice, this is usually chosen to be the surface of a sphere, or a rotational ellipsoid, three-axis ellipsoid, or the plane when a small portion of the surface is to be depicted neglecting the curvature. In this contribution, we choose as the reference surface of the Earth the surface of a sphere. On this surface, we shall use two types of coordinates.

- cartesian coordinates $(O, x, y, z)$ where $O$ is the origin of the reference surface and the z-axis corresponds to the Earth's axis.

- spherical coordinates $[r, \phi, \lambda]$, where $\phi$ is the latitude (northern, southern, calculated from the equator to the poles so that $\phi \in\left(-\frac{\pi}{2}, \frac{\pi}{2}\right)$ ) and $\lambda$ is the longitude (eastern, western, calculated from the plane defined by the Earth's axis and a fixed point - Greenwich - so that $\lambda \in\langle-\pi, \pi\rangle)$. The set of points on the reference surface with constant $\phi$ is called a parallel, and the set of points with constant $\lambda$ is a meridian. The system of meridians and parallels is called the cartographic grid.

If the prime meridian (with $\phi=0$ ) lies in the plane $(x, z)$, then the relationship between spherical $[r, \phi, \lambda]$ and cartesian $[x, y, z]$ coordinates is expressed as follows:

$$
\begin{aligned}
& x=r \cdot \cos \phi \cdot \cos \lambda, \\
& y=r \cdot \cos \phi \cdot \sin \lambda, \\
& z=r \cdot \sin \phi .
\end{aligned}
$$

\footnotetext{
* Darina Stachova

Department of Mathematics, Faculty of Science, University of Zilina, Slovakia, E-mail: darina.stachova@fpv.uniza.sk
} 


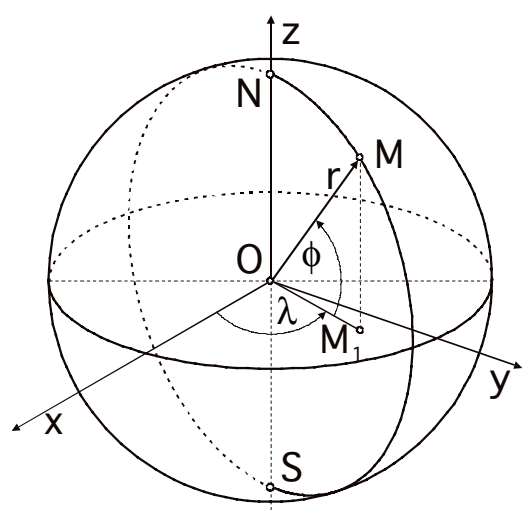

Fig. 1 Spherical coordinates on the reference surface

Cartographic projection is a system of methods and calculations transforming the cartographic grid to the plane of a map. Cartographic projection is defined by its transformation equations which in general are of the form $x=f(\phi, \lambda), y=g(\phi, \lambda)$. If each equation depends only on one coordinate of the reference surface, we call such projections simple projections which in their general form can be expressed as follows:

$$
x=n \cdot \lambda, y=g(\phi) .
$$

Projections can be expressed in cartesian coordinates, as is shown in the above example, or alternatively, in polar coordinates $(\varepsilon, \rho)$.

A separate group of projections form the so-called pseudoprojections. In these projections, one of the two transformation equations is a function of both coordinates of the reference surface. These include also the so-called polyconic projections which are essentially simple conical projections with an infinite number of projecting cones.

In total there are around 300 known types of cartographic projections out of which around 50 are simple; yet in practice only few of them are used. Since there are so many of them, we categorize the cartographic projections according to their common features such as the surface of projection, the position of the Earth's axis, the distortion of various geometric shapes such as angles, lengths of curves, areas of surfaces, etc.

According to the type of the surface of projection we distinguish the following types:

- azimuthal projection - mapping the reference surface to a planar surface

- cylindrical projection - mapping the reference surface to a cylindrical surface

- conical projection - mapping the reference surface to a conical surface

Projections that can be derived geometrically from the central projection are called map projections. In the case of azimuthal
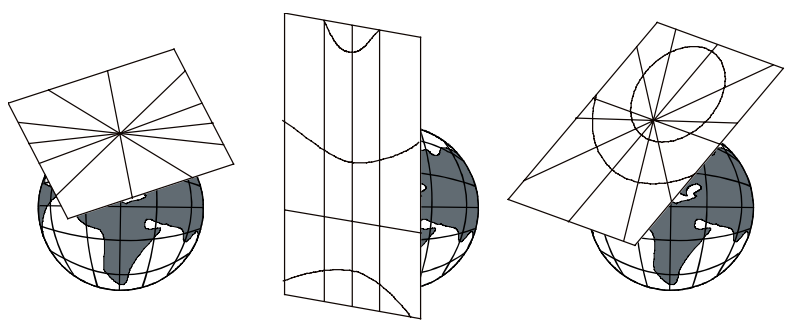

Fig. 2 Azimuthal projection
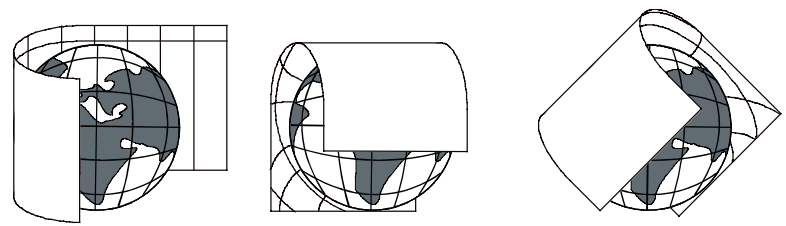

Fig. 3: Cylindrical projection
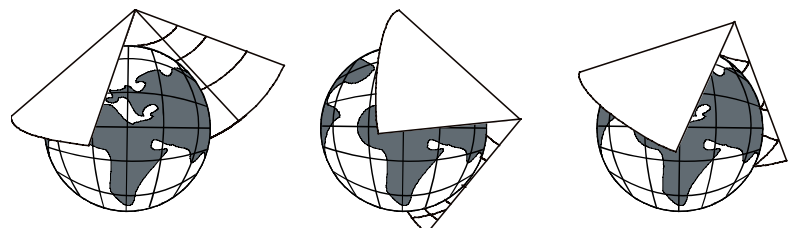

Fig. 4 Conical projection

map projections, depending on the position of the center, we further distinguish the following projections:

- gnomonic projection - the center of the projection is the center of the sphere; it is used for maps of polar regions and for naval maps. Its transformation equations are as follows:

$$
\varepsilon=\lambda \text { and } \rho=r \cdot \operatorname{tg} \delta
$$

where $\rho$ and $\varepsilon$ are polar coordinates on the map and $\delta=\frac{\pi}{2}-\phi$ is the zenith angle,

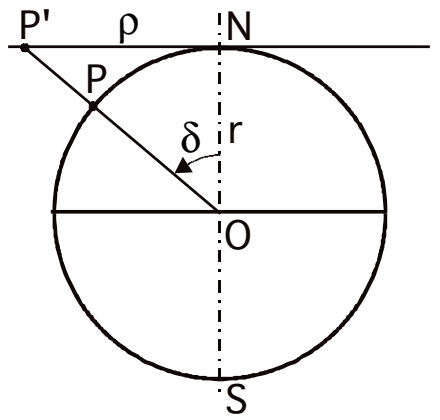

Fig. 5 Gnomonic projection 
- stereographic projection - the center of the projection lies on the pole; it depicts larger part of the surface than gnomonic projection and has the following transformation equations:

$$
\varepsilon=\lambda \text { and } \rho=2 r \cdot \operatorname{tg}(\delta / 2)
$$

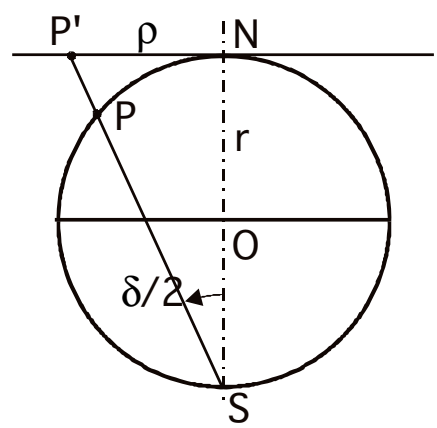

Fig. 6 Stereographic projection

- orthographic projection - the center of the projection is the point at infinity; the projection represents a view of the Earth from the outer space. Its transformation equations are as follows:

$\varepsilon=\lambda$ and $\rho=r \cdot \sin \delta$

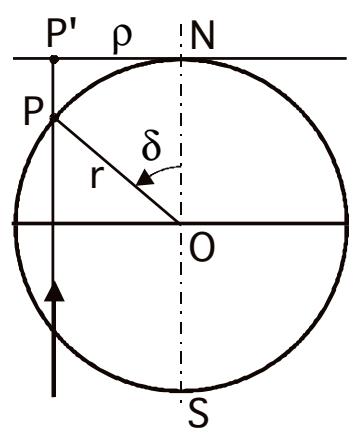

Fig. 7 Orthographic projection

- satellite projection - the center of the projection lies outside the Earth's surface; it is used for satellite imagery in weather forecast. Its transformation equations are as follows:

$$
\varepsilon=\lambda \text { and } \rho=\frac{(2 r+s) \cdot s \cdot \sin \delta}{r+s \cdot \cos \delta+s}
$$

- double projection - is a double stereographic projection; the point is first mapped to an auxiliary circle with radius $2 r$ and then it is stereographically projected to the plane. This projection introduces less distortion and has the following transformation equations:

$$
\varepsilon=\lambda \text { and } \rho=4 r \cdot \operatorname{tg}(\delta / 4)
$$

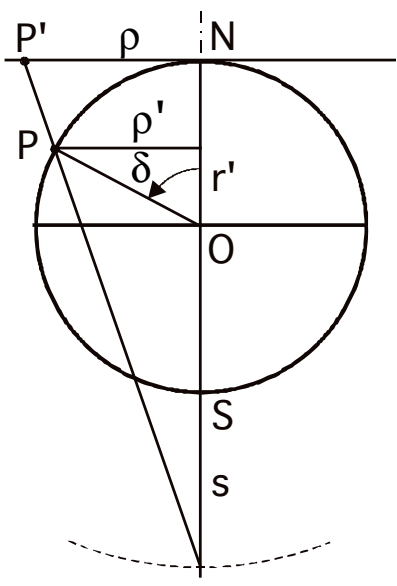

Fig. 8 Satellite projection

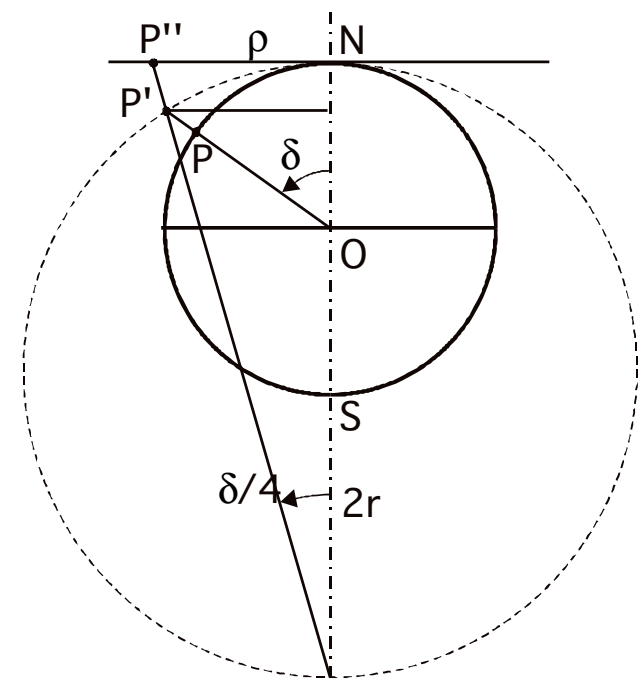

Fig. 9 Double projection

As we know from historical sources, the oldest known cartographic imagery is considered to be the geographic drawing of a settlement of hunter-gatherers from the region of Pavlov hills whose age is approximately 24000 years. Scientific foundations of cartography were established in the ancient Greece over a period of several centuries. During this time, geometry, physics, and other scientific disciplines were also founded in connection to the names such as Strabo, Euclid, Pythagoras, Archimedes, Erastothenes, Ptolemaios, Thales, and many others. Indeed, the word cartography does not have its Greek root by accident. Next significant impulse for development of cartography was the age of sea exploration and colonization in the years 1492-1522. For these reasons many cartographic projections are named after their inventors. For instance:

- azimuthal Lambert projection - Johan Geinrich Lambert (1772) - it is used for about $15 \%$ of all maps and is area-preserving. If the center of the projection is on the equator or in a general 
position, parallels and meridians are mapped to complicated curves. If the center is on the pole, the transformation equations are as follows:

$$
\varepsilon=\lambda \text { and } \rho=2 r \cdot \sin \delta
$$

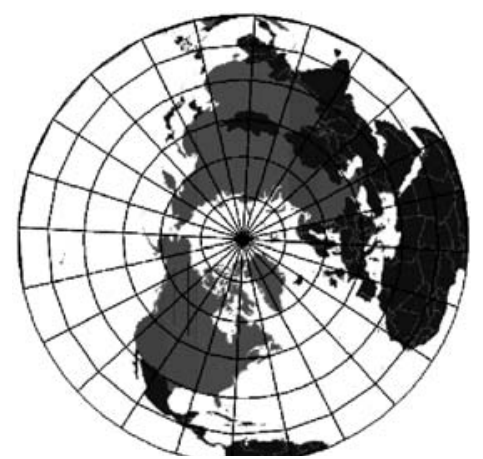

Fig. 10 Azimuthal Lambert projection with center on the pole

- azimuthal orthographic projection - Apollonius (third century B.C.) - the projection preserves lengths of parallels. If the center of the projection is on the equator, it maps meridians to elliptic arcs and parallels to parallel lines. In general position of the center, both meridians and parallels are represented as elliptic arcs. With the center on the pole, the transformation equations are of the form:

$$
\varepsilon=\lambda \text { and } \rho=r \cdot \sin \delta
$$

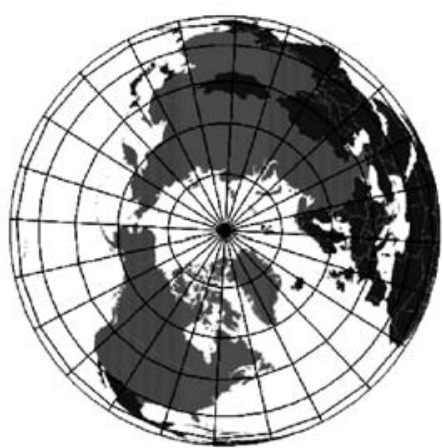

Fig. 11 Azimuthal orthographic projection with center on the pole

- Ptolemaic conical projection - Klaudios Ptolemaios (second century A.D.) - the projection preserves lengths of meridians and the length of the tangent parallel $\phi_{0}$. It is used for about $40 \%$ of maps in atlases using $\phi_{0}=45^{\circ}$. Its transformation equations are:

$$
\varepsilon=\lambda \cdot \cos \delta_{0} \text { and } \rho=r \cdot\left[\operatorname{tg} \delta_{0}-\left(\delta-\delta_{0}\right)\right]
$$

- Delisle conical projection - Joseph Nicholaus de l'Isle (1745) preserves the lengths of parallels $\phi_{1}$ and $\phi_{2}$ and lengths of all

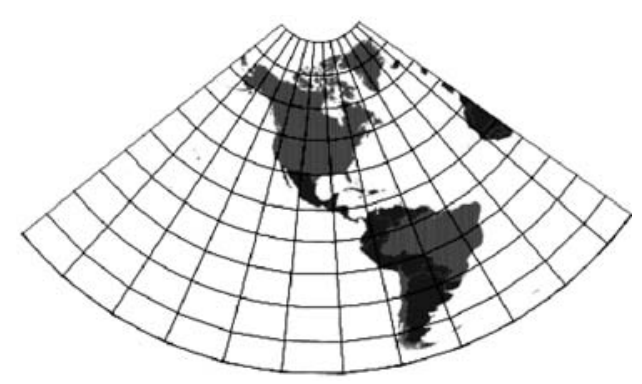

Fig. 12 Ptolemaic conical projection

meridians. Areas and angles are distorted less than with Ptolemaic projection. The transformation equations are:

$$
\begin{aligned}
& \varepsilon=\lambda \cdot \frac{\sin \delta_{1}-\sin \delta_{2}}{\delta_{1}-\delta_{2}} \text { and } \\
& \rho=r \cdot\left(\frac{\delta_{2} \cdot \sin \delta_{1}-\delta_{1} \cdot \sin \delta_{2}}{\sin \delta_{2}-\sin \delta_{1}}+\delta\right)
\end{aligned}
$$

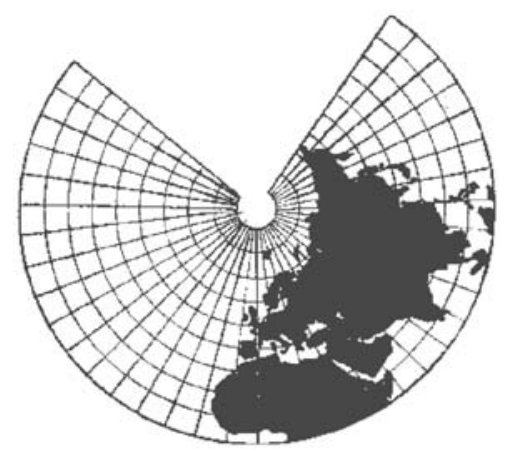

Fig. 13 Delisle conical projection

- Marinos' cylindrical projection - Marinos of Tyre (third century B.C.) - preserves lengths of meridians and parallels and introduces large distortion in the polar regions. Its transformation equations when expressed in spherical coordinates are as follows:

$$
x=r \cdot \lambda \text { and } y=r \cdot \varphi
$$

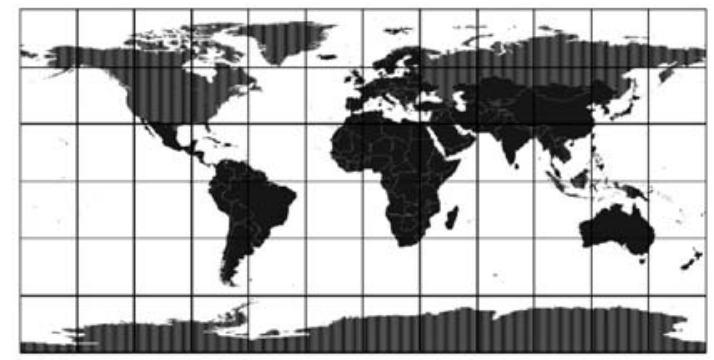

Fig. 14 Marinos' cylindrical projection 
- Mercator's cylindrical projection - Gerhard Mercator (1569) preserves angles and is used for navigation charts; it does not allow depicting the poles and it introduces large aerial distortion. Its transformation equations have the following form:

$$
x=r \cdot \lambda \text { and } y=r \cdot \ln (\operatorname{cotg}(\delta / 2)
$$

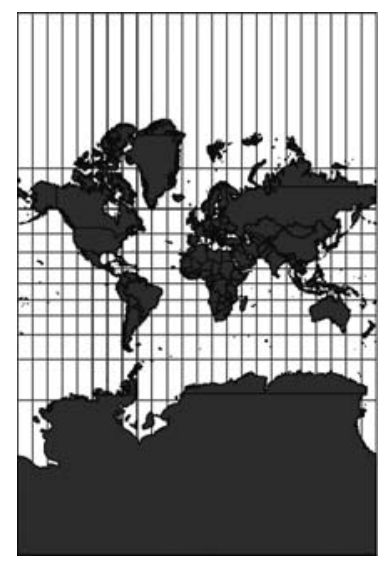

Fig. 15 Mercator's projection

- Mercator-Sanson's projection (also known as sinusoidal or SansonFlamsteed projection) - attributed to several authors - pseudocylindrical projection based on Marinos' projection; meridians are depicted as half sinusoids. It preserves lengths of parallels and of the prime meridian, both of which are mapped to line segments. Its transformation equations are of the form:

$$
x=r \cdot \lambda \cos \varphi \text { and } y=r \cdot \varphi
$$

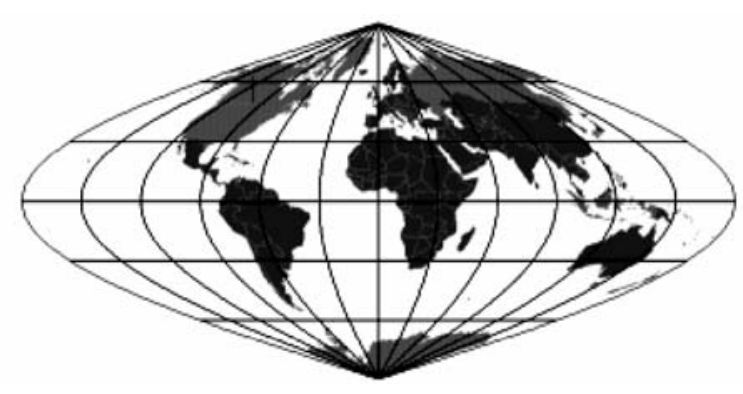

Fig. 16 Mercator-Sanson's projection

- Bonne's projection - Rigobert Bonne (1752) - pseudoconic projection formed from the Ptolemaic projection; it preserves lengths of parallels and of the prime meridian. It has the following transformation equations:

$$
\varepsilon=\frac{2 \pi \cdot \sin \delta}{\operatorname{tg} \delta_{0}+\delta-\delta_{0}} \text { and } \rho=r \cdot\left(\operatorname{tg} \delta_{0}+\delta-\delta_{0}\right) .
$$

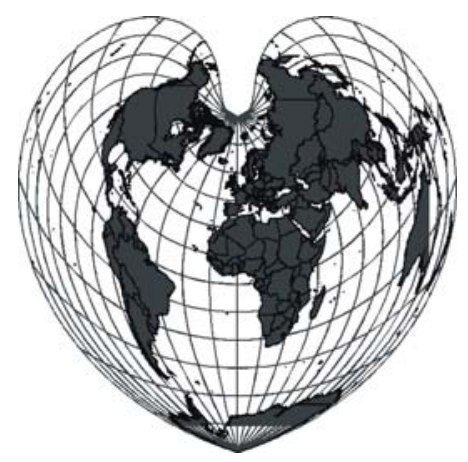

Fig. 17 Bonne's projection

- Hassler's polyconic projection - Ferdinand Rudolph Hassler (19th century) - the projection maps parallels to circular arcs of non-concentric circles and preserves lengths of parallels and of the prime meridian.

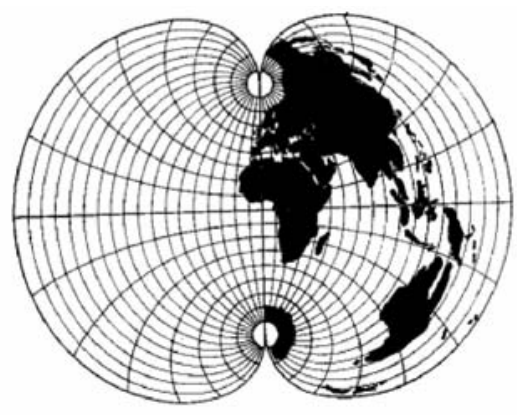

Fig. 18 Hassler's polyconic projection

\section{Conclusion}

The aim of this contribution was to acquaint the reader with cartographic projections, their categorization, and their precise mathematical description. Naturally, we do not present here a complete list of projections or aspects considered in their classification. We have focused mainly on those projections that we consider esthetically appealing and also those that are frequently used yet whose origin is not well known.

\section{Acknowledgement}

The author acknowledges support from the project KEGA SR 3/7090/09. 


\section{References}

[1] BARANOVA, M.: Mathematical Cartography (in Czech), manuscript, 2009, http://home.zcu.cz/ baranov/SPS_Kartografie/MatKart.pdf(accessed 09/09)

[2] DRABEK, K., HARANT, F., SETZER, O.: Descriptive Geometry - part II (in Czech), Praha, SNTL, 1979, ISBN 04-007-79

[3] KADERAVEK, F.: Geometry and Art in the Past (in Czech, Praha, Pudorys, 1997, ISBN 80-90791-5-6

[4] MEDEK, V., ZAMOZIK, J.: Constructive Geometry for Engineers (in Slovak), Bratislava, Alfa, 1978, ISBN 63-552-76

[5] TALHOFER, V.: Fundamentals of Mathematical Cartography (in Czech, Brno, Univerzita obrany, 2007, http://uzivatel.unob.cz/talhofer/Zaklady matematicke kartografie.pdf (accessed 09/09) ISBN 978-80-7231-297-9

[6] VAJSABloVA, M.: Cartographic Projections from the Perspective of Constructive Geometry (in Slovak), Zbornik zo seminara o pocitacovej geometrii SCG'99, Kocovce, 1999

[7] http://www.progonos.com/furuti/MapProj/CartIndex/cartIndex.html (accessed 09/09). 